\title{
Nível de ansiedade dos pacientes submetidos ao atendimento odontológico
}

Anxiety level of patients submitted to dental care

Nivel de ansiedad de los pacientes sometidos al atendimiento odontológico

Camila Helena Machado da Costa FIGUEIREDO ${ }^{1}$

Tuanny Lopes Alves Silvestre COURA ${ }^{2}$

Ocimar Lopes de OLIVEIRA ${ }^{3}$

Elizandra Silva da PENHA ${ }^{4}$

Luanna Abílio Diniz Melquíades de MEDEIROS

${ }^{1}$ Professora Doutora da Unidade Acadêmica de Ciências Biológicas, Centro de Saúde e Tecnologia Rural, Universidade Federal de Campina Grande (UFCG) 58700-970, Patos-Paraíba, Brasil

${ }^{2}$ Graduada em Odontologia, Unidade Acadêmica de Ciências Biológicas, Centro de Saúde e Tecnologia Rural, Universidade Federal de Campina Grande (UFCG) 58700-970, Patos-Paraíba, Brasil

${ }^{3}$ Graduando em Odontologia, Unidade Acadêmica de Ciências Biológicas, Centro de Saúde e Tecnologia Rural, Universidade Federal de Campina Grande (UFCG) 58700-970, Patos-Paraíba, Brasil

${ }^{4}$ Professora Doutora da Unidade Acadêmica de Ciências Biológicas, Centro de Saúde e Tecnologia Rural, Universidade Federal de Campina Grande (UFCG) 58700-970, Patos-Paraíba, Brasil

\section{Resumo}

A situação de tratamento odontológico em geral está relacionada à ansiedade e ao stress, assim, a dor, os aspectos fisiológicos e psicológicos envolvidos no tratamento podem ser avaliados pelos pacientes. Diante disso, o presente estudo tem como propósito avaliar o nível de ansiedade dos pacientes submetidos ao tratamento odontológico na Clínica Escola de Odontologia da Universidade Federal de Campina Grande, campus de Patos. Este estudo foi do tipo transversal, observacional, adotando como estratégia de coleta de dados o questionário específico. A amostra foi composta por 134 pacientes, a coleta de dados foi realizada por um pesquisador, que coletou as informações através de dois questionários. O primeiro questionário foi composto por questões de identificação (gênero, idade, frequência de consultas ao dentista e procedimento odontológico que causa desconforto). O segundo questionário por questões específicas na identificação do grau de ansiedade dos pacientes ao tratamento odontológico utilizando a escala DAS (Dental Anxiety Scale). Após coletados, os dados foram trabalhados pela estatística descritiva e submetidos ao teste estatístico Qui-quadrado $(\mathrm{p}<0,05)$. A prevalência de ansiedade foi de $87,3 \%$, sendo o nível moderado o mais prevalente $(39,6 \%)$. Dentre os procedimentos odontológicos que causam mais incômodo, o motor de alta rotação foi citado por $40,1 \%$ dos entrevistados. Ao associar a presença ou ausência da ansiedade com gênero, não foi encontrado diferença estatisticamente significativa ( $>00,05)$. Desta forma, conclui-se que a prevalência de ansiedade foi alta e a aquisição de um conhecimento mais profundo do sentimento de ansiedade são de extrema importância na execução dos procedimentos no consultório odontológico.

Descritores: Assistência Odontológica; Estresse Psicológico; Medicina do Comportamento.

\section{Abstract}

The situation of dental treatment is related in general to anxiety and stress, as well, the pain, the physiological and psychological aspects involved in the treatment can be assessed by patients. Thus, the present study aims to assess the level of anxiety of patients subjected to the dental treatment at the Clinical Dental School of the Federal University of Campina Grande, Patos campus. This study was cross-sectional, observational, adopting as data collection strategy the specific questionnaire. The sample was comprised of 134 patients and the data collection was done by a researcher who collected the information through two questionnaires. The first questionnaire was consisted of identification questions (gender, age, frequency of visits to the dentist and dental procedures that cause discomfort). In the second, were used specific questions to identify the degree of anxiety of patients during dental treatment using the DAS scale (Dental Anxiety Scale). Once collected, the data were worked by descriptive statistics and submitted to statistical Chi-square test ( $\mathrm{p}<0.05)$. The prevalence of anxiety was $87.3 \%$, the moderate level the most prevalent (39.6\%). Among the dental procedures that cause more uncomfortable, the high speed handpiece was cited by $40.1 \%$ of respondents. When associating the presence or absence of anxiety with gender, it was not found statistically significant difference ( $p>0.05)$. In this way, it was concluded that the prevalence of anxiety was high and the acquisition of a deeper knowledge of the feeling of anxiety is extremely important in the implementation of procedures in the dental office.

Descriptors: Dental Care; Stress, Psychological; Behavioral Medicine.

\section{Resumen}

La situación de tratamiento odontológico en general está relacionada con la ansiedad y el estrés, así, el dolor, los aspectos fisiológicos y psicológicos involucrados en el tratamiento pueden ser evaluados por los pacientes. Por ello, el presente estudio tiene como propósito evaluar el nivel de ansiedad de los pacientes sometidos al tratamiento odontológico en la Clínica Escuela de Odontología de la Universidad Federal de Campina Grande, campus de Patos. Este estudio fue del tipo transversal, observacional, adoptando como estrategia de recolección de datos el cuestionario específico. La muestra fue compuesta por 134 pacientes, la recolección de datos fue realizada por un investigador, que recogió las informaciones a través de dos cuestionarios. El primer cuestionario fue compuesto por cuestiones de identificación (género, edad, frecuencia de consultas al dentista y procedimiento odontológico que causa incomodidad). El segundo cuestionario por cuestiones específicas en la identificación del grado de ansiedad de los pacientes al tratamiento odontológico utilizando la escala DAS (Dental Anxiety Scale). Después de recolectados, los datos fueron trabajados por la estadística descriptiva y sometidos a la prueba estadística Qui-cuadrado ( $\mathrm{p}$ $<0,05)$. La prevalencia de ansiedad fue de $87,3 \%$, siendo el nivel moderado el más prevalente $(39,6 \%)$. Entre los procedimientos odontológicos que causan más incómodo, el motor de alta rotación fue citado por el 40,1\% de los entrevistados. Al asociar la presencia o ausencia de la ansiedad con género, no se encontró diferencia estadísticamente significativa ( $p>0,05)$. De esta forma, se concluye que la prevalencia de ansiedad fue alta y la adquisición de un conocimiento más profundo del sentimiento de ansiedad son de extrema importancia en la ejecución de los procedimientos en el consultorio odontológico.

Descriptores: Atención Odontológica; Estrés Psicológico; Medicina de la Conducta.

\section{INTRODUÇÃO}

Medo e ansiedade são sentimentos comuns a pacientes que necessitam de tratamento odontológico, representando uma barreira ao atendimento adequado do profissional ${ }^{1,2,3}$. O medo pode ser conceituado como um temor a algo ou alguma coisa que é externo e que se apresenta como um perigo real, que ameaça a integridade física ou psicológica da pessoa. $\mathrm{O}$ medo também é visto como um estado emocional de alerta ante o perigo, caracterizado por um conhecimento intelectual do mesmo, enquanto ansiedade está ligada a uma resposta emocional interna não facilmente reconhecível, com períodos de maior duração ${ }^{2,4}$. 
A ansiedade ao tratamento odontológico, geralmente, inicia-se na infância ou adolescência. Os principais fatores desencadeadores são: experiência dolorosa anterior, desconhecimento em relação aos procedimentos, o ambiente do consultório, idéias negativas repassadas por outras pessoas. Assim, a odontofobia atinge de 15 a $20 \%$ da população em geral $^{1}$.

Estudos verificaram que as mulheres são mais ansiosas durante o tratamento odontológico do que os homens ${ }^{2,3}$. Existem estudos indicando que os níveis de ansiedade são mais altos entre indivíduos com mais de 24 anos de idade ${ }^{5}$. Tratamentos odontológicos invasivos, como restaurações, extrações, procedimento protético de coroas/pontes, tratamento endodôntico e tratamento periodontal/cirurgia, foram associados com maior probabilidade de sentir dor durante o procedimento odontológico $^{6}$. Entre os procedimentos odontológicos, a injeção anestésica é um dos principais fatores na produção de fobia e ansiedade durante o tratamento ${ }^{2}$.

Desta forma, o presente estudo tem como propósito avaliar o nível de ansiedade dos pacientes submetidos ao tratamento odontológico na Clínica Escola de Odontologia da Universidade Federal de Campina Grande (UFCG).

\section{MATERIAL E MÉTODO}

Este estudo foi do tipo transversal, observacional, com abordagem indutiva $\mathrm{e}$ procedimento comparativo, estatístico-descritivo, como estratégia foi adotada a coleta de dados através de um questionário específico. $O$ universo foi composto pelos pacientes atendidos na disciplina de Clínica Multidisciplinar I e II do Curso de Odontologia da Universidade Federal de Campina Grande, campus de Patos. As disciplinas de Clínica Multidisciplinar I e II atendem as especialidades de dentística, periodontia, cirurgia e endodontia.

O cálculo amostral considerou um grau de confiança de $95 \%$, poder de teste de $50 \%$ e erro aceitável de 5\%, em um universo de 200 pacientes, obteve-se uma amostra de 134 participantes. O município foi selecionado por conveniência em função de ser o de maior porte populacional do Sertão Paraibano e a $3^{\text {a }}$ cidade polo do Estado da Paraíba, considerando sua importância socioeconômica. O município de Patos - Paraíba (PB) está localizado no sertão paraibano, distanciando-se da capital (João Pessoa) $298 \mathrm{~km}$ e possuindo 100.732 habitantes. O município, por sua situação geográfica no interior da Paraíba, se constitui num centro polarizador de uma vasta região interiorana do Estado, em torno do qual gravitam 50 municípios, e para o qual convergem os interesses de uma parcela bastante significativa da população. Este trabalho foi realizado na Universidade Federal de Campina Grande - instituição de Ensino Superior pública.
Para a participação dos pacientes nessa pesquisa foram considerados como critérios de inclusão: Ser paciente em atendimento na disciplina de Clínica Multidisciplinar I e/ou II da Clínica Escola do Curso de Odontologia da Universidade Federal de Campina Grande, campus de Patos; está presente na Clínica Escola no dia da coleta; apresentar mais de 18 anos; Autorização de participação da pesquisa por meio do Termo de Consentimento Livre e Esclarecido (TCLE) assinado.

Foram excluídos da pesquisa os pacientes que apresentem uma ou mais das seguintes características: Não for paciente em atendimento na disciplina de Clínica Multidisciplinar I e/ou II da Clínica Escola do Curso de Odontologia da Universidade Federal de Campina Grande, campus de Patos; não está presente na Clínica Escola no dia da coleta; apresentar menos de 18 anos; Ausência de autorização da participação no estudo. A coleta de dados foi realizada por um pesquisador, que coletou as informações através de dois questionários. O primeiro questionário continha questões de identificação, como gênero, idade, renda familiar, bem como frequência de consultas ao dentista e procedimento odontológico que causa desconforto. O segundo questionário era composto por questões específicas na identificação do grau de ansiedade dos pacientes ao tratamento odontológico utilizando a escala DAS (Dental Anxiety Scale). 7,8 Para a avaliação do grau de ansiedade, as respostas foram quantificadas, sendo $\mathrm{a}=1, \mathrm{~b}=2, \mathrm{c}=3, \mathrm{~d}=4, \mathrm{e}=5$. Conforme critério adotado por Corah (1969), 7 somando-se os valores atribuídos a cada questão, o intervalo possível de pontuação poderá variar, entre 4 e 20 pontos, sendo o nível de ansiedade classificado em nulo, baixo, moderado e exacerbado (Tabela 1).

Todos os voluntários participantes da pesquisa preencheram um Termo de Consentimento Livre e Esclarecido, por meio do qual foram informados dos objetivos do estudo e dos benefícios que este poderia trazer à população, bem como da possibilidade de abandono da pesquisa pelos mesmos em qualquer momento, sem nenhum ônus ao voluntário. O projeto de pesquisa foi enviado para o Comitê de Ética em Pesquisa com Seres Humanos da Faculdade Integrada de Patos (FIP) e aprovado sob o CAAE 48120815.8.0000.5181.Após coletadas, as informações foram registradas na forma de banco de dados do programa de informática SPSS (Statistical Package for Social Sciences) para Windows, versão 13.0, e trabalhadas pela estatística descritiva e submetidas ao teste estatístico Qui-quadrado considerado significativo ao nível de 5\% $(\mathrm{p}<0,05)$.

Tabela 1. Classificação do grau de ansiedade segundo a escala DAS $S^{7,8}$

\begin{tabular}{c|c}
\hline Pontuação & Grau de ansiedade \\
\hline 4 & Nulo \\
\hline 5 a 10 & Baixo \\
\hline 11 a 15 & Moderado \\
\hline 16 a 20 & Exacerbado \\
\hline
\end{tabular}




\section{RESULTADOS}

A amostra obteve 134 participantes, destes 71 (53\%) do gênero feminino e $63(47 \%)$ do gênero masculino. A idade dos participantes variou entre 18 e 81 anos, sendo a média de idade 37,5 anos.

Em relação ao estado civil, $66(49,3 \%)$ dos participantes eram solteiros, 57 (42,5\%) casados e 11 $(8,2 \%)$ divorciados. A renda familiar mensal variou entre $\mathrm{R} \$ 700,01$ até $\mathrm{R} \$ 1.500,00$ Reais para 71 pacientes $(53,0 \%)$, para $43(32,1 \%)$ foi inferior a $\mathrm{R} \$$ 700,00 Reais e para $18(13,4 \%)$ a renda foi superior a $\mathrm{R} \$ 1.500,01$ Reais.

Quanto à busca pelos serviços odontológicos, $57(42,5 \%)$ pacientes consultou o cirurgião-dentista a última vez entre 1 ou 2 anos, $57(42,5 \%)$ menos de um ano, $16(11,9 \%)$ três anos ou mais e $4(3,0 \%)$ participantes não lembraram a última vez da consulta.

Dentre os procedimentos odontológicos que causaram mais incômodo, o motor de alta rotação foi citado por $40,1 \%$ dos entrevistados, seguido da anestesia $31,7 \%$ e cirurgia $14,1 \%$ (Tabela 2). Para a identificação do grau de ansiedade, $87,3 \%$ dos pacientes demonstraram algum grau de ansiedade, sendo o nível moderado o mais prevalente $(39,6 \%)$ (Tabela 3). A Tabela 4 aponta os resultados das perguntas do questionário na identificação do grau de ansiedade. Ao associar a presença ou ausência da ansiedade com gênero, não foi encontrado diferença estatisticamente significativa $(\mathrm{p}>0,05)$ (Tabela 5).

Tabela 2. Distribuição da amostra segundo o procedimento odontológico de maior incômodo

\begin{tabular}{|c|c|c|c|}
\hline \multirow{5}{*}{$\begin{array}{l}\text { Procedimento } \\
\text { odontológico de } \\
\text { maior incômodo* }\end{array}$} & Variáveis & $\mathbf{n}$ & $\%$ \\
\hline & Alta rotação & 77 & 40,1 \\
\hline & Anestesia & 61 & 31,7 \\
\hline & Cirurgia & 27 & 14,1 \\
\hline & Nenhum & 27 & 14,1 \\
\hline \multicolumn{4}{|c|}{ * $\overline{\text { Considerando que alguns entrevistados expressaram mais de uma resposta. }}$} \\
\hline \multicolumn{4}{|c|}{ Tabela 3. Distribuição dos pacientes segundo o nível de ansiedade } \\
\hline \multirow{5}{*}{$\begin{array}{l}\text { Escala de ansiedade } \\
\text { dentária (DAS) }\end{array}$} & Variáveis & $\mathbf{n}$ & $\%$ \\
\hline & Nulo & 17 & 12,7 \\
\hline & Baixo & 50 & 37,3 \\
\hline & Moderado & 53 & 39,6 \\
\hline & Exacerbado & 14 & 10,4 \\
\hline Total & & 134 & 100 \\
\hline
\end{tabular}

Tabela 4. Distribuição da amostra segundo o questionário de identificação do grau de ansiedade

\begin{tabular}{|c|c|c|c|}
\hline Perguntas DAS & Variáveis & $\mathbf{n}$ & $\%$ \\
\hline \multirow{5}{*}{$\begin{array}{c}\text { Se você tivesse } \\
\text { que ir ao } \\
\text { dentista } \\
\text { amanhã, como } \\
\text { você se } \\
\text { sentiria? }\end{array}$} & $\begin{array}{l}\text { Eu estaria antecipando uma experiência } \\
\text { razoavelmente agradável }\end{array}$ & 38 & 28,4 \\
\hline & Eu não me importaria & 42 & 31,3 \\
\hline & Eu me sentiria ligeiramente desconfortável & 21 & 15,7 \\
\hline & $\begin{array}{l}\text { Eu temo que eu me sentiria desconfortável } \\
\text { e teria dor }\end{array}$ & 17 & 12,7 \\
\hline & $\begin{array}{l}\text { Eu estaria com muito medo com que o } \\
\text { dentista me fizesse }\end{array}$ & 16 & 11,9 \\
\hline \multirow{5}{*}{$\begin{array}{l}\text { Quando você } \\
\text { está esperando } \\
\text { na sala de } \\
\text { espera do } \\
\text { dentista, como } \\
\text { você se sente? }\end{array}$} & Relaxado & 46 & 34,3 \\
\hline & Meio desconfortável & 9 & 6,7 \\
\hline & Tenso & 35 & 26,1 \\
\hline & Ansioso & 39 & 29,1 \\
\hline & $\begin{array}{c}\text { Tão ansioso que começo a suar ou começo } \\
\text { a me sentir mal }\end{array}$ & 5 & 3,7 \\
\hline \multirow{5}{*}{$\begin{array}{c}\text { Antes do } \\
\text { atendimento } \\
\text { com a turbina, } \\
\text { como você se } \\
\text { sente? }\end{array}$} & Relaxado & 38 & 28,4 \\
\hline & Meio desconfortável & 23 & 17,2 \\
\hline & Tenso & 38 & 28,4 \\
\hline & Ansioso & 26 & 19,4 \\
\hline & $\begin{array}{l}\text { Tão ansioso que começo a suar ou começo } \\
\text { a me sentir mal }\end{array}$ & 9 & 6,7 \\
\hline \multirow{5}{*}{$\begin{array}{c}\text { Antes do } \\
\text { atendimento } \\
\text { com } \\
\text { instrumentos } \\
\text { periodontais, } \\
\text { como você se } \\
\text { sente? }\end{array}$} & Relaxado & 40 & 29,9 \\
\hline & Meio desconfortável & 26 & 19,4 \\
\hline & Tenso & 42 & 31,3 \\
\hline & Ansioso & 20 & 14,9 \\
\hline & $\begin{array}{c}\text { Tão ansioso que começo a suar ou começo } \\
\text { a me sentir mal }\end{array}$ & 6 & 4,5 \\
\hline
\end{tabular}

Tabela 5. Associação entre gênero e presença/ausência de ansiedade

\begin{tabular}{ccccc}
\hline \multirow{2}{*}{ Gênero } & \multicolumn{3}{c}{ Ansiedade } & P \\
\cline { 2 - 3 } & Sim & Não & \\
\hline Feminino & 62 & 9 & \multirow{2}{*}{$0,997^{\mathrm{a}}$} \\
\hline Masculino & 55 & 8 &
\end{tabular}

\section{DISCUSSÃO}

O medo pode ser definido como um sentimento de grande inquietação diante de um perigo real, de uma ameaça, susto, pavor, temor ou terror. Pode-se nomear esta sensação muito comum entre os pacientes diante da realização de algum procedimento odontológico como Odontofobia. A fobia, então, é um medo irracional percebido pelo próprio indivíduo como excessivo e sem proporção, mas que, quando diante da circunstância temida, não consegue deixar de sentir?

Já a ansiedade diante dos procedimentos odontológicos é o que Maniglia-Ferreira et. al. ${ }^{10}$ chamaram de ansiedade odontológica, o que a nosso ver é um empecilho para prevenções muitas vezes necessárias, que poderiam evitar maiores danos à saúde bucal do paciente.

Com relação aos métodos para avaliar a ansiedade, segundo Newton e Buck, a escala Corah, é a mais utilizada na literatura científica por esta apresentar boa consistência interna e confiabilidade teste-reteste e foi traduzida ao português por Pereira et al. .

Foi constatado diante da pesquisa que os principais agentes causadores da ansiedade em atendimento de rotina foram o motor de alta rotação e a anestesia. Há uma concordância com o trabalho de Pereira et al. ${ }^{4}$ e Bottan et al. ${ }^{1}$ que também encontraram como gerador de ansiedade a anestesia e motor de alta rotação, enquanto que no estudo de Chaves et al. ${ }^{11}$ a prevalência foi do motor de alta rotação e a realização de cirurgias.

Deste modo, a ansiedade pode apresentar reações físicas, que por sua vez tanto pode impossibilitar o inicio de um tratamento odontológico a medida em que muitas pessoas que precisam de fato de um cuidado odontológico evitam os consultórios ou procuram um serviço odontológico apenas em casos de extrema necessidade ${ }^{8}$.

Segundo Maniglia-Ferreira et. al. ${ }^{10}$, os pacientes que apresentam algum grau de ansiedade evitam frequentemente o tratamento odontológico, independente do seu nível social ou escolaridade.

No presente trabalho, os resultados em relação à ansiedade estão de acordo com os resultados encontrados por Chaves et al. ${ }^{11}$, Bottan et al. ${ }^{1}$ e Pereira et al. ${ }^{4}$ onde a prevalência de ansiedade foi alta. Todavia, Siviero et al. ${ }^{3}$ encontraram em sua pesquisa uma prevalência de ansiedade baixa.

$\mathrm{Na}$ associação do gênero com presença/ausência da ansiedade Chaves et al. ${ }^{11}$, Bottan et al. ${ }^{1}$ e Siviero et al. ${ }^{3}$, verificaram associação diferente da encontrada no nosso estudo, no qual as 
mulheres apresentaram maior grau de ansiedade do que os homens. Já Medeiros et al. ${ }^{2}$, Maniglia-Ferreira et al. ${ }^{10}$, Pereira et al. ${ }^{4}$ e Santos et al. ${ }^{9}$ apresentaram resultados semelhantes, não encontrando associação significativa entre gênero e nível de ansiedade odontológica.

Podemos perceber que mesmo com tecnologia aliada à Odontologia ainda não foi possível produzir instrumentos que causem menor desconforto ao paciente, uma vez que a anestesia e o uso do motor de alta rotação são essenciais para realização de grande parte dos procedimentos.

Nesse contexto de atendimento dos pacientes com algum grau de ansiedade, é sugerido que os profissionais exerçam um papel fundamental ante $o$ tratamento odontológico através de programas preventivos e curativos na busca de uma melhor interação e envolvimento do paciente no processo de sua manutenção da saúde bucal.

\section{CONCLUSÃO}

De acordo com os resultados obtidos, podemos concluir que a prevalência da ansiedade ao tratamento odontológico foi alta sendo que de acordo com o questionário de avaliação do grau de ansiedade, o nível moderado foi o mais prevalente entre os participantes. Ao associar a ansiedade com o gênero não foi encontrada diferença estatisticamente significativa.

Assim, a ansiedade odontológica é uma característica peculiar que varia de acordo com cada paciente e a aquisição de um conhecimento mais profundo do sentimento de ansiedade por parte do profissional com a inserção de campanhas e programas preventivos repassados com explicação simples ao alcance do entendimento dos pacientes, pode desmistificar a ansiedade e o medo frente ao atendimento odontológico.

\section{REFERÊNCIAS}

1. Bottan ER, Oglio JD, Araújo SM. Ansiedade ao Tratamento Odontológico em Estudantes do Ensino Fundamental. Pesq Bras Odontoped Clin Integr. 2007;7(3):241-46.

2. Medeiros LA, Ramiro FMS, Lima CAA, Souza LMA, Fortes TMV, Gropp FC. Avaliação do grau de ansiedade dos pacientes antes de cirurgias orais menores. Rev Odontol UNESP. 2013;42(5):357-63.

3. Siviero M, Nhani VT, Prado EFGB. Análise da ansiedade como fator preditor de dor aguda em pacientes submetidos à exodontias ambulatoriais. Rev Odontol UNESP. 2008;37(4):329-36.

4. Pereira VZ, Barreto RC, Pereira GAS, Cavalcanti HRBB. Avaliação dos níveis de ansiedade em pacientes submetidos ao tratamento odontológico. R bras ci Saúde. 2013;17(1):55-64.

5. Udoye CI, Oginni AO, Oginni FO. Dental anxiety among patients undergoing various dental treatments in a nigerian teaching hospital. J Contemp Dent Pract. 2005;6(2):91-8.

6. Maggirias J, Locker D. Psychological factors and perceptions of pain associated with dental treatment. Community Dent Oral Epidemiol. 2002;30(2):151-59.

7. Corah NL. Development of a dental anxiety scale. J Dent Res. 1969;48(4):596.

8. Pereira LHMC, Ramos DLP, Crosato E. Ansiedade e dor em odontologia - enfoque psicofisiopatológico. Rev Assoc Paul Cir Dent. 1995;49(4):285-90.

9. Santos PAD, Campos JADB, Martins CS. Avaliação do sentimento de ansiedade frente ao atendimento odontológico. Rev Uniara. 2007; 20:189-202.

10. Maniglia-Ferreira C, Gurgel-Filho ED, Böneckervalverde G, Moura EH, Deus G, Coutinho-Filho T. Ansiedade odontológica: nível, prevalência e comportamento. RBPS. 2004; 17(2):51-5.

11. Chaves AM, Loffredo LCM, Valsecki-Júnior A, Chavez OM, Campos JADB. Estudo epidemiológico da ansiedade dos pacientes ao tratamento odontológico. Rev Odontol UNESP. 2006;35(4):263-68.

\section{CONFLITO DE INTERESSES}

Os autores declaram não haver conflitos de interesse.

\section{AUTOR PARA CORRESPONDÊNCIA}

\section{Camila Helena Machado da Costa Figueiredo}

Unidade Acadêmica de Ciências Biológicas, Centro de Saúde e Tecnologia Rural, Universidade Federal de Campina Grande Rua Severino Soares, S/N, Condomínio Villas do Lago, Q.14, L.11, Maternidade, 58701380, Patos - Paraíba, Brasil e-mail: camila_helena_@hotmail.com

Submetido em 09/08/2019 Aceito em 20/04/2020 